\title{
Estado nutricional e níveis hematológicos e séricos de ferro em pré-escolares de municípios com diferentes índices de desenvolvimento infantil
}

\author{
Nutritional status, hematological and serum levels of iron in preschool children from cities with different \\ child development indexes
}

\section{Wilson Luis de Oliveira' ${ }^{1}$, Fernanda Luisa C. Oliveira², Olga Maria S. Amancio ${ }^{3}$}

RESUMO

Objetivo: Avaliar o estado nutricional e os níveis hematológicos e séricos de ferro em pré-escolares de municípios com diferentes índices de desenvolvimento infantil (IDI).

Métodos: Estudo transversal de 34 pré-escolares com idade de três a seis anos do município de Laranjal, Paraná, com IDI baixo e com o mesmo número de crianças pareadas por idade e gênero, provenientes de Presidente Prudente, São Paulo, município com IDI médio. Avaliou-se o estado nutricional por meio do escore $Z$ das relações peso/estatura e estatura/idade. A avaliação bioquímica constou de dosagens de hemoglobina, hematócrito, ferro sérico e ferritina. Para a análise estatística foram usados os testes $t$ de Student, Mann-Whitney, quiquadrado e o exato de Fisher.

Resultados: Peso, estatura e escore $Z$ das relações antropométricas foram significantemente menores no grupo de crianças de Laranjal comparados aos de Presidente Prudente. A prevalência de anemia em ambos os grupos foi $8,8 \%$, não havendo diferença significante para a prevalência de ferropenia e para os valores de ferritina sérica.

Conclusões: Pré-escolares do município com IDI baixo apresentam mais desnutrição, enquanto sobrepeso e obesidade são predominantes nos pré-escolares do município com IDI médio. Quanto às alterações dos níveis hematológicos e séricos de ferro, não há diferença entre os pré-escolares dos municípios estudados.

Palavras-chaves: estado nutricional; ferro; pré-escolar.

\section{ABSTRACT}

Objective: To evaluate nutritional status and iron hematological and serum levels of preschool children from cities with different child development indexes (CDI).

Methods: This cross-sectional study enrolled 34 preschool children aged between three to six years from two municipalities of Brazil: Laranjal, Paraná, with a low CDI, and Presidente Prudente, Sao Paulo, with a medium CDI. Children of both cities were matched by age and gender. The nutritional status was evaluated by $\mathrm{Z}$ score for weight/height and height/age. The biochemical evaluation included hemoglobin, hematocrit, seric iron and ferritin levels. The following tests were used for statistical analysis: $t$ test, Mann-Whitney, chi-square and Fisher's exact test.

Results: The weight, height and $\mathrm{Z}$ score of the anthropometric relationships were significantly lower in the group of Laranjal children. Anemia was present in $8.8 \%$ of the children studied in both cities, without significant differences in the prevalence of iron deficiency or in the ferritin levels.

Conclusions: The preschool children from the city with low CDI presented mainly malnutrition, while overweight and obesity were more prevalent on preschool children from the city with a medium CDI. Hematimetric values, iron and ferritin levels were similar between the preschool children of both cities.

Key-words: nutritional status; iron; child preschool.
${ }^{1}$ Mestre em Ciências pela Universidade Federal de São Paulo (Unifesp), São Paulo, SP, Brasil.

2Doutora em Medicina pela Universidade Federal de São Paulo (Unifesp), São Paulo, SP, Brasil

${ }^{3}$ Doutora em ciências aplicadas à Pediatria, professora adjunta da Unifesp, São Paulo, SP, Brasil
Endereço para correspondência:

Olga Maria Silverio Amancio

Rua Botucatu, 703

CEP 04023-062 - São Paulo/SP

E-mail: omsamancio.dped@epm.br

Recebido em: 14/12/2007

Aprovado em: 8/5/2008

Fonte financiadora: Unoeste 


\section{Introdução}

Nas últimas décadas, a concepção de desenvolvimento e bem-estar da população de um país ultrapassa dimensões tradicionais de renda pessoal e crescimento econômico. Desde 1990, o Programa das Nações Unidas para o Desenvolvimento (PNUD) vem promovendo o paradigma do desenvolvimento humano sustentável a partir de três atributos básicos de desenvolvimento: das pessoas, para as pessoas e pelas pessoas.

$\mathrm{O}$ índice de desenvolvimento humano (IDH) ${ }^{(1)}$, criado para acompanhar, nos países das Nações Unidas, os avanços no processo global de desenvolvimento humano, permite aferir longevidade de uma população, medida pela esperança de vida ao nascer, educação, taxas de alfabetização e de matrícula no ensino fundamental, médio e superior, além da renda, ajustada pela paridade de compra entre os países.

Com o intuito de contribuir para o desenvolvimento humano das crianças e dos adolescentes brasileiros, o Fundo das Nações Unidas para Infância (Unicef) ${ }^{(2)}$ desenvolveu o índice de desenvolvimento infantil (IDI), que incorpora doutrinas da Convenção sobre os Direitos da Criança e do Estatuto da Criança e do Adolescente, dando especial ênfase ao cuidado integral na primeira infância para a sobrevivência, crescimento e desenvolvimento (CISCD).

O IDI de um município varia de zero a um, sendo um o valor máximo que o município deveria buscar atingir no processo de sobrevivência, crescimento e desenvolvimento de suas crianças no primeiro período de vida. O IDI é classificado como elevado se acima de 0,800, médio entre 0,500 e 0,799 e, baixo, quando menor de 0,500. O IDI e seus indicadores são considerados pontos de partida para a análise dos problemas da primeira infância, levando-se em conta a própria natureza da criança e seu desenvolvimento.

A faixa pré-escolar se caracteriza por ser uma fase na qual, apesar de haver lenta velocidade de crescimento, a oferta adequada de macro e micronutrientes propiciará repleção, que será utilizada para a última fase de rápido crescimento.

Dentre os micronutrientes, destaca-se o ferro por sua função no crescimento e desenvolvimento, por meio da sua atuação no transporte e armazenamento de oxigênio, reações de liberação de energia, co-fator de reações enzimáticas e inúmeras outras ${ }^{(3)}$. A anemia por deficiência de ferro representa um dos maiores problemas nutricionais da população mundial, independentemente de idade e gênero, estimando-se que 66 a $80 \%$ dela seja deficiente em ferro e, mais de $30 \%$, anêmica ${ }^{(4)}$.
Além dos fatores fisiológicos, nutricionais e infecciosos ${ }^{(5)}$, devem ser lembrados como agentes agravantes, e muitas vezes determinantes da deficiência de ferro, o baixo nível socioeconômico e cultural, as condições de saneamento básico e de acesso aos serviços de saúde.

É importante o diagnóstico precoce da anemia ferropriva, pois esse estado carencial determina distúrbios no crescimento e no desenvolvimento neuropsicomotor da criança de intensidade variável, no comportamento cognitivo, além de estar associado ao aumento da suscetibilidade às infecções ${ }^{(5,6)}$.

Atualmente, vive-se em um meio em que a prevalência de anemia ferropriva depende de características regionais, observando-se nas últimas décadas aumento significativo de prevalência e gravidade em todas as regiões do país, independentemente do nível econômico ${ }^{(7)}$.

O crescimento e o desenvolvimento de crianças sofrem influências do meio ambiente, que refletem os aspectos socioeconômicos, psicológicos e nutricionais ${ }^{(8)}$. Na literatura, há associação de distúrbios nutricionais com o baixo nível socioeconômico ${ }^{(9,10)}$. Considerando-se o IDI de um município como ferramenta de avaliação do crescimento e desenvolvimento da primeira infância, justifica-se a realização deste trabalho que tem como objetivo avaliar o estado nutricional, os valores hematimétricos, os níveis séricos de ferro e ferritina de pré-escolares de um município com baixo IDI, comparados aos valores obtidos em pré-escolares de município com IDI médio.

\section{Métodos}

Estudo transversal realizado com 34 pré-escolares, com idade de três a seis anos, de ambos os gêneros, matriculados na creche municipal de Laranjal, Paraná, com IDI baixo $(0,263)^{(2)}$ e 1.510 habitantes na área urbana (167 crianças de três a seis anos de idade). A economia voltada é para a agricultura, pecuária e comércio. Estudou-se o mesmo número de crianças, pareadas por idade e gênero, matriculadas na creche municipal de Presidente Prudente, São Paulo. Presidente Prudente é um município com IDI médio $(0,747)^{(2)}$, que conta com 185.229 habitantes na área urbana (11.992 crianças de três a seis anos) e sua economia se baseia na agricultura, pecuária e comércio. O trabalho foi aprovado pelo Comitê de Ética em Pesquisa da Universidade Federal de São Paulo, havendo necessidade de prévia autorização por escrito dos pais ou responsáveis para a participação no estudo. Houve também a aprovação por escrito dos responsáveis das creches envolvidas.

O único critério de inclusão na pesquisa foi a faixa etária de entre três e seis anos. Foram excluídas as crianças portadoras 
de doença crônica, infecções agudas virais ou bacterianas, avaliadas por médico pediatra, aquelas hospitalizadas nos últimos 30 dias antes da pesquisa e as que faziam uso de suplemento nutricional de ferro. Formaram-se dois grupos: IDI baixo e IDI médio

Para obter a casuística, a prefeitura de Laranjal convocou duas vezes, com alto falante pelas ruas do município, a presença das crianças para, em data marcada, serem avaliadas. Compareceram 114 crianças de três a dez anos, das quais 34 foram selecionadas, formando-se assim uma amostra por adesão.

$O$ peso e a estatura foram obtidos de acordo com técnicas padronizadas ${ }^{(11)}$. O cálculo do escore $Z$ das relações peso/ estatura (P/E) e estatura/idade (E/I) foi feito pelo software Epi-Info 2000, versão 3.3.2, que utiliza como referência, dados do Center for Disease Control and Prevention ${ }^{(12)}$.

Em relação ao escore $Z$ de $P / E$, classificou-se em desnutrido $(<-2)$, risco para desnutrição (entre -2 e -1 ), peso adequado $(-1$ e +1$)$, sobrepeso $(+1$ e +2$)$ e obeso $(>+2)$. Quanto ao escore $Z$ da E/I, classificou-se em baixa estatura $(<-2)$, risco para baixa estatura (entre -2 e -1 ), estatura adequada (-1 e $+2)$ e alta estatura $(>+2)^{(13)}$.

As amostras de sangue foram coletadas entre $7 \mathrm{~h} 30$ e $10 \mathrm{~h}$, após jejum de oito horas. $\mathrm{O}$ soro foi estocado a $-18^{\circ} \mathrm{C}$ até o momento das dosagens, exceto para a hemoglobina e o hematócrito, que foram determinados imediatamente após a coleta. Todos os reagentes foram de grau analítico e os materiais do laboratório lavados com ácido nítrico.

Para determinar a hemoglobina, foi utilizado o método da cianometahemoglobina e, para o hematócrito, o do microematócrito. Considerou-se a presença de anemia se: hemoglobina $<1,0 \mathrm{~g} / \mathrm{dL}$ e hematócrito $<33 \%$ para crianças abaixo de cinco anos e hemoglobina $<11,5 \mathrm{~g} / \mathrm{dL}$ e hematócrito $<4 \%$ para crianças de cinco a seis anos ${ }^{(14)}$.
Para o ferro sérico, foi utilizado o Ferene-S kit Iron ${ }^{\circledR}$ da Bayer e, para a ferritina, Kit Immulite-Ferritin ${ }^{\circledR}$ da Diagnostic Products Corporation, que utiliza ensaio imunométrico. Os valores de referência adotados foram ferro sérico $<56 \mathrm{mcg} /$ $\mathrm{dL}^{(15)}$; ferritina sérica $<12 \mathrm{ng} / \mathrm{mL}$ (crianças abaixo de cinco anos) ou $<15 \mathrm{ng} / \mathrm{mL}$ (crianças acima de cinco anos) ${ }^{(14)}$.

$\mathrm{Na}$ análise estatística, utilizou-se o teste $t$ de Student para amostras independentes para comparar as variáveis paramétricas entre os dois grupos, o teste de Mann-Whitney para comparar as variáveis não paramétricas e o qui-quadrado ou teste exato de Fischer para estudar as associações das variáveis categóricas entre os grupos. Adotou-se $p<0,05$ como significante.

\section{Resultados}

Os dois grupos foram homogêneos em relação à idade $(p=0,97)$ e gênero. Entretanto, o grupo com IDI baixo apresentou peso corporal, estatura e, consequentemente, escore $Z$ das relações $\mathrm{P} / \mathrm{E}$ e E/I significantemente menores $(p=0,0001)$ em relação ao grupo IDI médio (Tabela 1).

Observaram-se distúrbios nutricionais com características diferentes entre os grupos. Só houve ocorrência de crianças com desnutrição, risco para desnutrição e baixa estatura no grupo com IDI baixo. Por outro lado, a presença de sobrepeso e obesidade foi de $50 \%$ no grupo com IDI médio e $8,8 \%$ no grupo IDI baixo (Tabela 2).

Os resultados médios da hemoglobina, hematócrito, ferro e ferritina estavam de acordo com os valores de referência adotados e não mostraram diferença significante entre os grupos, com exceção do hematócrito que foi maior no grupo com IDI baixo $(p=0,006)$, apesar de ambos estarem no intervalo de normalidade (Tabela 3).

Tabela 1 - Características e escore Z das relações peso para a estatura e estatura para a idade dos pré-escolares das cidades com IDI baixo e médio

\begin{tabular}{lccc}
\hline & \multicolumn{2}{c}{ Pré-escolares } & \multirow{2}{*}{ Valor de $\boldsymbol{p}$} \\
\cline { 2 - 3 } & \multicolumn{1}{c}{ IDI baixo $(\mathbf{n = 3 4 )}$} & IDI médio $(\mathbf{n = 3 4 )}$ & 0,97 \\
\cline { 2 - 3 } Idade (anos) & $4,9 \pm 0,8(3,2$ a 6$)$ & $4,9 \pm 0,8(3$ a 6$)$ & \\
Masculino:Feminino & $18: 16$ & $18: 16$ & 0,0001 \\
Peso $(\mathrm{kg})$ & $15,6 \pm 2,9(10,7$ a 27,5$)$ & $21,9 \pm 4,5(15,4$ a 33,8) & 0,0001 \\
Estatura(cm) & $103,5 \pm 7,8(80$ a 122$)$ & $111,4 \pm 7,6(97$ a 132) & 0,0001 \\
Escore Z (peso/estatura) & $-0,76 \pm 1,23(-3,22$ a 3,22) & $1,40 \pm 1,90(-0,91$ a 7,38$)$ & 0,0001 \\
Escore Z (estatura/idade) & $-1,05 \pm 1,27(-5,41$ a 2,03$)$ & $0,66 \pm 1,14(-1,96$ a 4,05$)$ & \\
\hline
\end{tabular}


Tabela 2 - Distribuição dos pré-escolares dos grupos com IDI baixo e médio de acordo com o estado nutricional avaliado pelo escore $Z$ das relações peso para a estatura e estatura para a idade

\begin{tabular}{lcc}
\hline & \multicolumn{2}{c}{ Pré-escolares } \\
\cline { 2 - 3 } & $\begin{array}{c}\text { IDI baixo } \\
\mathbf{n}(\%) / \text { variação }\end{array}$ & $\begin{array}{c}\text { IDI médio } \\
\mathbf{n}(\%) / \text { variação }\end{array}$ \\
\hline Peso/Estatura & & - \\
<-2 (desnutrido) & $4(11,8 \%) /-3,22$ a $-2,12$ & - \\
Escore Z entre -2 e -1 (risco para desnutrição) & $11(32,3 \%) /-1,85$ a $-1,04$ \\
Escore Z entre -1 e +1 (peso adequado) & $16(47,1 \%) /-0,97$ a 0,99 & $17(50 \%) /-0,91$ a 0,92 \\
Escore Z entre 1 e +2 (sobrepeso) & $2(5,9 \%) / 1,31$ a 1,48 & $9(26,5 \%) / 1,01$ a 1,83 \\
$>+2$ (obeso) & $1(2,9 \%) / 3,22$ & $8(23,5 \%) / 2,05$ a 7,38 \\
Estatura/Idade & & - \\
<-2 (baixa estatura) & $7(20,6 \%) /-5,41$ a $-2,03$ & $2(5,9 \%) /-1,96$ a $-1,08$ \\
Escore Z entre -2 e -1 (risco para baixa estatura) & $9(26,5 \%) /-1,59$ a $-1,14$ & $30(88,2 \%) /-0,96$ a 1,92 \\
Escore Z entre -1 e 2 (estatura adequada) & $17(50 \%) /-0,94$ a 0,86 & $2(5,9 \%) / 2,06$ a 4,05 \\
$>+2$ (alta estatura) & $1(2,9 \%) /$ & \\
\hline
\end{tabular}

( )variação dos valores encontrados.

Tabela 3 - Valores médios de hemoglobina, hematócrito, ferro sérico e ferritina dos pré-escolares dos grupos com IDI baixo e médio

\begin{tabular}{lccl}
\hline & \multicolumn{2}{c}{ Pré-escolares } & \multirow{2}{*}{ Valor de $\boldsymbol{p}$} \\
\cline { 2 - 3 } & IDI baixo $(\mathrm{n}=34)$ & IDI médio $(\mathrm{n}=34)$ & \\
\hline Hemoglobina $(\mathrm{g} / \mathrm{dL})$ & $12,6 \pm 0,97(10,7$ a 14,3$)$ & $12,2 \pm 0,82(11$ a 14,2$)$ & 0,09 \\
Hematócrito $(\%)$ & $39,1 \pm 2,5(35$ a 45$)$ & $37,5 \pm 2,1(35$ a 42$)$ & 0,006 \\
Ferro sérico $(\mathrm{mcg} / \mathrm{dL})$ & $59,6 \pm 28,8(11$ a 125$)$ & $65,4 \pm 25,6(21$ a 116$)$ & 0,45 \\
Ferritina sérica $(\mathrm{ng} / \mathrm{mL})$ & $34,3 \pm 21,1(3,3$ a 91$)$ & $36,4 \pm 19,8(3$ a 88,9$)$ & 0,42 \\
\hline
\end{tabular}

A prevalência de anemia foi semelhante $(8,8 \%)$ nos dois grupos. A ferropenia foi encontrada em $15 / 34(44,1 \%)$ dos pré-ecolares do grupo com IDI baixo e em $13 / 34(38,2 \%)$ dos pré-escolares do grupo com IDI médio, não havendo diferença entre os grupos. Em relação à ferritina, cinco $(14,7 \%)$ e quatro $(11,8 \%)$ dos pré-escolares dos grupos com IDI baixo e médio, respectivamente, estavam com valores inadequados segundo a referência, não havendo diferença entre os grupos, conforme Tabela 4.

\section{Discussão}

A área da saúde da Universidade do Oeste Paulista (Unoeste) rotineiramente realiza levantamentos visando, se necessário, futuros programas de intervenção nos municípios de Laranjal e Presidente Prudente. Em Laranjal, por se tratar de um município pequeno, é praxe que a prefeitura faça a convocação por meio de alto-falante, à qual a população responde bem. Assim, o comparecimento de 110 crianças na creche (única na cidade), das quais 34 com a idade pretendida para a pesquisa, caracteriza, além da adesão, uma amostra de conveniência. Entende-se, portanto, que os resultados referem-se somente à população estudada.

Vários fatores ambientais interferem no adequado crescimento e desenvolvimento desde a fase intra-uterina até a adolescência $^{(16)}$. Assim, no município com baixo IDI haveria condições que resultariam principalmente em desnutrição, risco para desnutrição e baixa estatura, cristalizando-se a idéia de que a desnutrição está relacionada com condições ambientais precárias, com baixo nível socioeconômico, deficit de saneamento básico, baixa escolaridade, estimulação pobre, desajustamento familiar, baixo peso ao nascimento e ausência ou abandono precoce do aleitamento materno, entre outras ${ }^{(16)}$. Algumas destas variáveis compõem ou estão intimamente 
Tabela 4 - Distribuição dos pré-escolares dos grupos com IDI baixo e médio de acordo com os valores adequados de hemoglobina, hematócrito, ferro e ferritina

\begin{tabular}{|c|c|c|c|c|c|c|c|}
\hline & & \multicolumn{4}{|c|}{ Pré-escolares } & & \\
\hline & & \multicolumn{2}{|c|}{ IDI baixo (n=34) } & \multicolumn{2}{|c|}{ IDI médio (n=34) } & \multicolumn{2}{|c|}{ Total $(n=68)$} \\
\hline & & $\mathbf{n}$ & $\%$ & $\mathbf{n}$ & $\%$ & $\mathbf{n}$ & $\%$ \\
\hline \multirow[t]{2}{*}{ Anemia $^{1}$} & $\operatorname{Sim}$ & 3 & 8,8 & 3 & 8,8 & 6 & 8,8 \\
\hline & Não & 31 & 91,2 & 31 & 91,2 & 62 & 91,2 \\
\hline \multirow[t]{2}{*}{ Hematócrito } & Adequado & 34 & 100,0 & 34 & 100,0 & 68 & 100,0 \\
\hline & Inadequado & - & - & - & - & - & - \\
\hline \multirow[t]{2}{*}{ Ferropenia $^{2}$} & Sim & 15 & 44,1 & 13 & 38,2 & 28 & 41,2 \\
\hline & Não & 19 & 55,9 & 21 & 61,8 & 40 & 58,8 \\
\hline \multirow[t]{2}{*}{ Ferritina $^{3}$} & Adequado & 29 & 85,3 & 30 & 88,2 & 59 & 86,8 \\
\hline & Inadequado & 5 & 14,7 & 4 & 11,8 & 9 & 13,2 \\
\hline
\end{tabular}

$1-p=1 ; 2-p=0,62 ; 3-p=0,72$

relacionadas ao IDI, explicando os resultados encontrados no grupo com IDI baixo.

Estudo que comparou os índices antropométricos obtidos em dois inquéritos nacionais (em 1989, por meio da Pesquisa Nacional de Saúde e Nutrição e, em 1996, pela Pesquisa Nacional sobre Demografia e Saúde), em crianças menores de cinco anos, revela diminuição da prevalência de desnutrição (escore $\mathrm{Z}$ de $\mathrm{P} / \mathrm{E}<-2$ ) de 6,8 para 5,7\%, com maior prevalência de desnutrição nas regiões Norte e Nordeste e nas mães com menor escolaridade. Esta comparação mostra, ainda, que a prevalência de baixa estatura para crianças menores de cinco anos, segundo o escore $Z$ de E/I, diminuiu de 15,1 para $10,5 \%$, com redução na região Nordeste de 27,1 para 17,9\%. Quando avaliadas as crianças maiores de dois anos, não se observou diferença significativa entre os dois inquéritos, indicando que a diminuição da prevalência de deficits deve-se às crianças até dois anos de vida ${ }^{(17)}$. Outro estudo, com 536 crianças menores de 11 anos, em uma comunidade de Recife, detectou retardo de crescimento linear (E/I) em 5\% das crianças estudadas. Destas, $204(41,7 \%)$ eram préescolares de dois a seis anos incompletos ${ }^{(10)}$.

Sem dúvida, o comprometimento estatural no pré-escolar indica agravos nutricionais pregressos por fatores genéticos ou ambientais. A desnutrição intra-uterina e/ou pós-natal, desencadeada por privação nutricional, aliada à ausência ou interrupção precoce do aleitamento materno, às infecções de repetição no primeiro ano de vida, ao baixo nível socioeconômico e às más condições de saneamento básico, pode acarretar maior risco de baixa estatura nos pré-escolares do município com baixo IDI. Por outro lado, a melhora da qualidade de vida, gerando facilidades e confortos da vida urbana, situações estas relacionadas ao IDI médio, levaria a condições mais apropriadas para o crescimento, mas facilitaria também o aparecimento de sobrepeso e obesidade, como observado no grupo com IDI médio, cuja prevalência de obesidade foi maior que a descrita no Sul $(6,7 \%)^{(18)}$ e Nordeste $(8,5 \%)^{(19)}$ do país.

De acordo com a Organização Mundial da Saúde, a prevalência de obesidade infantil tem crescido de 10 a $40 \%$ na maioria dos países europeus nos últimos dez anos ${ }^{(20)}$. Quanto à comparação entre os dois inquéritos nacionais anteriormente mencionados, não houve diferença significativa na prevalência de obesidade (escore $Z$ de $\mathrm{P} / \mathrm{E}>+2$ ), que foi de 5,5 e 4,9\%. Porém, observou-se diminuição da prevalência de obesidade nas regiões Sudeste e Sul do Brasil, com aumento na região Nordeste, assim como redução significativa nas mães com escolaridade maior e discreto incremento nas mães com menor escolaridade ${ }^{(17)}$. No caso específico da escolaridade materna, esta é importante para a saúde da criança, uma vez que o maior nível de conhecimento formal parece influenciar as práticas relacionadas aos cuidados infantil ${ }^{(21)}$.

O Brasil, assim como vários países em desenvolvimento, apresenta-se em fase de transição nutricional, mostrando, de um lado, bolsões de pobreza com predomínio de desnutrição energético-protéica e, de outro, áreas em que a pequena melhoria das condições socioeconômicas está desencadeando aumento da freqüência de sobrepeso e obesidade.

No presente estudo, a prevalência de anemia foi menor do que a verificada em pré-escolares de outras regiões brasileiras. Na cidade de São Paulo, em pré-escolares de zero a cinco anos, constatou-se anemia em 35,6\% ${ }^{(22)}$. Em Recife, Pernambuco, em crianças de seis meses a cinco anos de idade, 
detectou-se anemia em 28,3 e 55,1\%, em dois serviços públicos de saúde ${ }^{(23)}$. No agreste pernambucano, a prevalência de anemia foi de $38,9 \%{ }^{(24)}$. Houve associação significante em crianças com idade inferior a 24 meses de anemia e baixa renda, demonstrando que o fator alimentar estava associado à presença de anemia ${ }^{(25)}$.

O estudo dos fatores causais da anemia em dois inquéritos relata que o nível socioeconômico, a renda familiar, a escolaridade familiar, o saneamento básico e o acesso à saúde não apresentavam associação significativa com a anemia ${ }^{(7)}$. Confirmando esses resultados, o presente estudo não mostrou diferença na prevalência de anemia das crianças dos municípios com diferentes IDIs.

A presença de estoque adequado de ferro garante a manutenção do seu nível sérico. Infecções e inflamações crônicas e/ou ausência de estoque de ferro por ingestão insuficiente podem acarretar sua diminuição, o que ocorreu

\section{Referências bibliográficas}

1. United Nations Development Program [homepage on the internet]. The Human development index [cited 2008 Mar 18]. Available from: http://hdr.undp.org/en/ statistics/indices/hdi

2. UNICEF Brazil [homepage on the internet]. Situação da infância brasileira 2001: O índice de desenvolvimento infantil-IDI [cited 2005 Mar 17]. Available from: http://www.unicef.org/brazil

3. Aggett PJ. Physiology and metabolism of essential trace elements: an outline. Clin Endocrinol Metab 1985; 14:513-43.

4. World Health Organization [homepage on the internet]. Battling iron deficiency anaemia. The challenge [cited 2005 Jun 26]. Available from: http://whqlibdoc. who.int/hq/2000/WHO NHD 00.7.pdf

5. Andrews NC, Bridges KP. Disorders of iron metabolism and sideroblastic anaemia. In: Nathan DG, Oski FA, editors. Nathan \& Oski's hematology of infancy and childhood. $5^{\text {th }}$ ed. Philadelphia: Saunders; 1998. p. 423-62.

6. Lozoff B, De Andraca I, Castillo M, Smith JB, Walter T, Pino P. Behavioral and developmental effects of preventing iron-deficiency anemia in healthy full-term infants. Pediatrics 2003;112:846-54.

7. Monteiro CA, Szarfarc SC. Secular trends in childhood in the city of São Paulo, Brazil (1984-1996). Rev Saude Publica 2000;34 (6 Suppl):13-8.

8. Campos AL, Nascimento CFL, Grazini JT, de Assis AN, Vítolo MR, Nóbrega FJ. Aspectos nutricionais, psicológicos e sociais de mães de crianças desnutridas. J Pediatr 1995;71:214-8.

9. Sawaya AL. Desnutrição energético-protéica. In: Sawaya AL, editor. Desnutrição urbana no Brasil em um período de transição. São Paulo: Cortez; 1997. p. 19-33.

10. Motta MEFA, Silva GAP. Malnutrition and obesity in children: describing the profile of a low-income community. J Pediatr 2001;77:288-93.

11. Jelliffe DB. Evaluación del estado de nutrición de la comunidad. Geneve, Switzerland: WHO; 1968.

12. Centers for Disease Control and Prevention [homepage on the Internet]. 2000 CDC Growth Charts for the United States: methods and development. Series Report 11, no 246 [cited 2005 Apr 18]. Available from: http://www.cdc.gov/nchs/ data/series/sr_11/sr11_246.pdf

13. World Health Organization. Physical status: the use and interpretantion of anthropometry. Technical Reports Series n 854. Geneva: WHO;1995. em 41,2\% dos pré-escolares analisados, indicando que, apesar da baixa prevalência de anemia nos dois municípios, a disponibilidade de ferro não se encontrava adequada em quase metade das crianças. A prevalência de $13,6 \%$ de deficiência de ferro é descrita em crianças francesas saudáveis de dois a seis anos de idade ${ }^{(26)}$. Encontrou-se ainda, no estudo aqui apresentado, prevalência de deficiência de ferritina em $13,2 \%$ do total de pré-escolares, valor este menor do que o anteriormente descrito em pré-escolares brasileiros $(24,2 \%)^{(27)}$.

Os resultados obtidos permitem concluir que pré-escolares do município com IDI baixo apresentam predominantemente desnutrição, enquanto que sobrepeso e obesidade são predominantes nos pré-escolares do município com IDI médio. Quanto às alterações dos níveis hematológicos e séricos de ferro, não há diferença entre os pré-escolares dos municípios estudados.
14. World Health Organization. Iron deficiency anaemia: assessment, prevention, and control. A guide for programme managers. Geneve: WHO; 2001.

15. Franco CD. Ferritin. In: Kaplan LA, Pesce AJ, editors. Methods in clinical chemistry. St Louis: Mosby; 1980. p. 1240-2.

16. Nóbrega FJ. Evolução a longo prazo do desnutrido grave. Pediatr Mod 1997;33:143-66

17. Taddei JAAC, Colugnati FAB, Rodrigues EM, Sigulem DM, Lopez FA. Desvios nutricionais em menores de cinco anos. São Paulo: Unifesp; 2002.

18. Post CL, Victora CG, Barros FC, Horta BL, Guimarães PRV. Desnutrição e obesidade infantis em duas coortes de base populacional no Sul do Brasil: tendências e diferenciais. Cad Saude Publ 1996;12 (Suppl 1):S49-57.

19. Balaban G, Silva GAP. Prevalência de sobrepeso e obesidade em crianças e adolescentes de uma escola da rede privada de Recife. J Pediatr 2001;77:96-100.

20. Mello ED, Luft VC, Meyer F. Obesidade infantil: como podemos ser eficazes? J Pediatr 2004;80:173-82.

21. Wachs TD, Creed-Kanashiro H, Cueto S, Jacoby E. Maternal education and intelligence predict offspring diet and nutritional status. J Nutr 2005;135:2179-86.

22. Monteiro CA, Szarfarc SC. Estudo das condições de saúde das crianças no município de São Paulo, SP (Brasil), 1984-1985. Rev Saude Publ 1987;21: 255-60.

23. Salzano AC, Torre MAA, Batista Filho M, Romani SAM. Anemia em crianças de dois serviços de saúde de Recife-PE (Brasil). Rev Saude Publ 1985;19:499-507.

24. Lira PI, Cartagena HA, Romani SAM, Torres MA, Batista Filho M. Estado nutricional de crianças menores de seis anos, segundo posse da terra, em áreas rurais do Estado de Pernambuco, Nordeste do Brasil. Arch Latinoam Nutr 1985;35:247-57.

25. Sigulem DM, Tudisco ES, Paiva ER, Guerra CC. Anemia nutricional e parasitose intestinal em menores de 5 anos. Rev Paul Med 1985;103:308-12.

26. Preziosi P, Hercberg S, Galan P, Devanlay M, Cherouvrier F, Dupin H. Iron status of a healthy French population: factors determining biochemical markers. Ann Nutr Metabol 1994;38:192-202.

27. Almeida CA, Ricco RG, Ciampo LA, Souza AM, Pinho AP, Oliveira JE. Fatores associados a anemia por deficiência de ferro em crianças pré-escolares brasileiras. J Pediatr 2004:80:229-34. 